\title{
Potencial cariogênico de medicamentos, fármacos e drogas: Uma revisão
}

\author{
Cariogenic potential of medicines, medications and drugs: A review \\ Potencial cariogénico de medicamentos, fármacos y drogas: Una revisión
}

Recebido: 14/03/2021 | Revisado: 21/03/2021 | Aceito: 24/03/2021 | Publicado: 02/04/2021

Acácio Costa Silva
ORCID: https://orcid.org/0000-0001-7814-6681
Cristo Faculdade do Piauí, Brasil
E-mail: acaciocosta989@ gmail.com
Mauro Gustavo Amaral Brito
Cristo Faculdade do Piauí, Brasil
ORCID: https://orcid.org/0000-0002-9551-4025
E-mail: drmaurogustavo@ hotmail.com
Gabriel Mauriz de Moura Rocha
ORCID: https://orcid.org/0000-0003-1454-0414
Cristo Faculdade do Piauí, Brasil
E-mail: mauriz45@ hotmail.com
Mônica do Amaral Silva
Cristo Faculdade do Piauí, Brasil
ORCID: https://orcid.org/000-0001-6234-275X
E-mail: monica.amaral83@gmail.com
Luís Mário Rezende Júnior
Cristo Faculdade do Piaú, Brasil
ORCID: https://orcid.org/0000-0002-1145-9744
E-mail: profmariorezende@gmail.com
Guilherme Antônio Lopes de Oliveira
ORCID: https://orcid.org/0000-0003-3820-0502
Cristo Faculdade do Piauí, Brasil
E-mail: guilhermelopes@live.com

\section{Resumo}

Objetivo: Caracterizar a etiologia da cárie dentária, citando agentes com potencial cariogênico esclarecendo os mecanismos envolvidos, e ações no sentido de prevenir o desenvolvimento da mesma. Metodologia: Foi realizado revisão bibliográfica nas bases de dados: Scientific Electronic Library Online (SciElo), Google Acadêmico e PubMed. Sendo selecionados artigos, monografias, dissertações e teses, datados entre 2005 a 2020, nos idiomas português e inglês. A busca foi realizada por meio dos descritores: medicamentos (medication), drogas (drugs), fisiopatologia (phatophsiology) e cárie dentária (dental caries). E os artigos duplicados, incompletos foram excluídos da pesquisa. Resultados: Tendo o conceito e etiologia da cárie dentária esclarecido, aprofundou-se o conhecimento sobre o potencial cariogênico de medicamentos, fármacos e drogas, e os mecanismos envolvidos, evidenciando amplas ações, que dependem de situações especificas para se evidencia a cárie de medicamento, como resultado. Além de se observar que a principal prevenção se dar através do repasse de informações através de uma equipe multidisciplinar. Conclusão: Sugere-se que as substâncias aqui abordadas apresentam potencial cariogênico seja pela presença de açúcares fermentáveis na sua composição, ou pelo seu efeito adverso, xerostomia, e ainda ação corrosiva direta sobre os dentes.

Palavras-chave: Cáries; Etiologia; Fármacos; Medicamentos; Xerostomia.

\begin{abstract}
Objective: to characterize the etiology of dental caries, citing agents with cariogenic potential, clarifying the involved mechanisms and actions to prevent their development. Methodology: A bibliographic review was carried out on the basis of data: Scientific Electronic Library Online (SciElo), Google Scholar and PubMed. Articles, monographs, dissertations and thesis were selected, closed between 2005 and 2020, in Portuguese and English. The search was carried out through descriptors: medicines, drugs, physiopathology and dental caries. Duplicate and incomplete articles were excluded from research. Results: once the concept and etiology of dental caries has been clarified, knowledge about the cariogenic potential of drugs, drugs and drugs, and the involved, deepened mechanisms, evidencing broad actions, which depend on specific situations to reveal it caries of drugs, the consecuencia. Also note that the main prevention is through the transfer of information through a multidisciplinary team. Conclusion: It is suggested that the substances discussed here have cariogenic potential due to the presence of fermentable sugars in their composition, due to their adverse effect, xerostomy and even direct corrosive action on the teeth.
\end{abstract}

Keywords: Caries; Etiology; Drugs; Medication; Xerostomy. 


\begin{abstract}
Resumen
Objetivo: caracterizar la etiología de la caries dental, citando agentes con potencial cariogénico, aclarando los mecanismos involucrados y las acciones para prevenir su desarrollo. Metodología: Se realizó una revisión bibliográfica en las bases de datos: Scientific Electronic Library Online (SciElo), Google Scholar y PubMed. Se seleccionaron artículos, monografías, disertaciones y tesis, fechados entre 2005 y 2020, en portugués e inglés. La búsqueda se realizó a través de los descriptores: medicamentos, drogas, fisiopatología y caries dental. Y los artículos duplicados e incompletos fueron excluidos de la investigación. Resultados: una vez aclarado el concepto y la etiología de la caries dental, el conocimiento sobre el potencial cariogénico de los medicamentos, las drogas y las drogas, y los mecanismos involucrados, profundizados, evidencian acciones amplias, que dependen de situaciones específicas para revelar la caries de drogas, a consecuencia. Además de observar que la prevención principal es a través de la transferencia de información a través de un equipo multidisciplinario. Conclusión: Se sugiere que las sustancias discutidas aquí tienen potencial cariogénico debido a la presencia de azúcares fermentables en su composición, o debido a su efecto adverso, xerostomía e incluso acción corrosiva directa sobre los dientes.
\end{abstract}

Palabras clave: Caries; Etiología; Fármaco; Medicamento; Xerostomía.

\title{
1. Introdução
}

A cavidade oral é a região anatômica humana com a maior variedade de microrganismos, composta principalmente por bactérias e fungos, e não raramente por vírus. Esses microrganismos vivem em um biossistema que, quando se encontra em equilíbrio, sustenta a saúde oral. Essa área consiste em várias estruturas, cada uma representando um nicho ou ambiente que propicia o desenvolvimento de microrganismos. Com isso cita-se que esse órgão pode e, é exposto a inúmeros elementos físicos, químicos e biológicos. Sendo capaz de sofrer constantemente alterações que comprometam a homeostase oral do organismo (Silva, 2016).

Sendo o exemplo de enfermidade comum que acomete a extensão oral, a cárie dentária, doença multifatorial, infecciosa, transmissível e dieta dependente. Considerada a enfermidade crônica mais corriqueira em todo o mundo e que afeta todos os habitantes e de todas as faixas etárias. Um processo anormal, que causa perda dentária e dor na cavidade oral, e que envolve conjuntos de elementos onde ocorre desequilíbrio e reequilíbrio, retratado pelos episódios de desmineralização e remineralização fora das condições naturais (Campos et al., 2006).

Desse modo o conhecimento sobre o mecanismo fisiopatológico, a etiologia em si da cárie dentária, envolve muito mais do que elementos que influenciavam a fisiologia da cavidade bucal em condições especificas. Mais também a real função da biodiversidade microbiológica, habitual e patogênica, a cronicidade de exposição a agentes e a susceptibilidade individual do hospedeiro, com todos esses elementos inseridos num processo complexo, ocasionando o problema (Lima, 2007).

Nesse contexto, apresenta-se os fármacos, medicamentos e drogas, substâncias com capacidade de alteração orgânica fisiológica e patológica, podendo estas desencadearem efeitos tóxicos, adverso ou colaterais em organismos consumidores. Expressando-se que algumas classes podem se torna fatores de risco para o desenvolvimento da cárie, levando em consideração a frequência de administração, ou seja, a cronicidade, a forma farmacêutica utilizada e/ou a sua toxidade. A cárie é manifestada como um efeito indesejável provocada pela administração rotineira destes químicos pelo paciente, principalmente no caso de doenças crônicas ou abuso de substâncias (Passos; Freitas; Sampaio, 2008; Bittencourt; Caponi; Maluf, 2013).

Os prováveis mecanismos por qual esses químicos atuam ocasionando a cárie de medicamento são, o feito xerostomizante (diminuição da liberação de saliva), visto a importante função do fluxo salivar na prevenção da cárie. Sendo que os principais fármacos citados em literatura são os agonistas beta-adrenérgicos e anti-histamínicos. Já o outro mecanismo, é atribuído a forma farmacêutica do produto, destacando as soluções e suspensões com altas concentrações de açúcares, baixo pH e elevada acidez, onde se sobressair os medicamentos pediátricos. No entanto, as literaturas são controvérsias quanto ao potencial cariogênico dos fármacos, medicamentos e drogas. Havendo relatos específicos de mecanismos próprios para indução de cáries dependendo da substância (Freitas et al., 2016; Leal et al., 2015). 
Apesar de se encontrar na literatura trabalhos com essa temática, esses estudos ainda são escassos. Desse modo o presente estudo tem o objetivo de caracterizar a etiologia da cárie dentária, citando os principais agentes registrados em bibliografia, com potencial cariogênico esclarecendo os mecanismos envolvidos, e ações no sentido de prevenir o desenvolvimento da mesma.

\section{Metodologia}

A pesquisa realizada é uma revisão bibliográfica, do tipo qualitativa e descritiva, a qual foi realizada busca de dados bibliográficos nas bases científicas: Scientific Electronic Library Online (SciElo), Google Acadêmico e PubMed. Foram selecionados artigos publicados entre 2005 a 2020, nos idiomas português e inglês. Como critérios de inclusão para o trabalho, apenas artigos completos e que melhores se enquadrarem na temática abordada. E exclusos da pesquisa, os artigos incompletos, os que não estiveram dentro da margem temporal pré-estabelecida e os duplicados. A busca foi norteada pela utilização dos termos: medicamentos (medication), drogas (drugs), fisiopatologia (phatophsiology) e cárie dentaria (dental caries). Com isso, analisou-se pesquisas clínicas, artigos de revisão, monografias, dissertações e teses. As palavras-chaves selecionadas estão de acordo com DeCS (Descritores em Ciências da Saúde).

\section{Revisão da literatura}

\subsection{Conceito e etiologia da cárie dentária}

Sabe-se que a etiologia da cárie dentária envolve fatores que não podem ser reproduzidos fora das condições naturais. E que se relaciona a inúmeros fenômenos em um processo complexo. O título mais aceito para conceituar a cárie dentária envolve um processo patológico multifatorial, infecciosa, transmissível e dieta dependente. Mais de maneira simples o mecanismo que se relaciona com a sua causa envolve a perca de minerais dos dentes devido à ação de ácidos produzidos por fermentação (Freitas, 2015; Lima, 2007).

Além de quer, qualquer estimulo que induza redução do pH oral e que chegue ao contato da superfície dos dentes, de maneira rotineira, oferece risco de eliminar a hidroxiapatita $\left(\mathrm{Ca}_{10}\left(\mathrm{PO}_{4}\right) 6 \mathrm{OH}_{2}\right)$, originando perda de tecido mineralizado, e consequente erosão. Visto que quando o pH está em torno de 5,5, o ambiente torna-se crítico para hidroxiapatita, pois esta é solúvel em meios ácidos (Gomes \& Silva, 2010).

Esse pH pode-se reduzido, como já citado pela ação de microrganismo, através da fermentação. Sendo o de maior destaque nas bibliografias, a espécie Streptococcus mutans (microrganismo acidogénicos e acidófilo), que através dos hidratos de carbono, produz ácidos como o acético, láctico, fórmico e propiónico no biofilme dentário, levando a lesão cariosa, que é patognomônico da doença cárie (Freitas et al., 2016).

$\mathrm{Na}$ faixa de $\mathrm{pH}$ reduzido ocorre processo fisiológico a fim de manter equilíbrio estrutural do esmalte do dente, esse denominado des-remineralização, esta atuação, juntamente com participação da saliva (efeito tampão), busca regular a perda e o ganho do mineral (Gomes \& Silva, 2010; Freitas, 2015).

Em consequência há essa infinidade de fatores, é que a etiologia da cárie, possui sua complexidade, tornando-se muito difícil compreendê-la completamente. Por esse enredo, ainda em meados de 1969, Keyes, desenvolveu um modelo etiológico ou macro-tendência multicausal-biologicista, que é conhecido até os dias atuais, e atenciosamente denominado "tríade de Keyes". E mais posteriormente, Newbrum o aperfeiçoou, levando em consideração o fator tempo, ou o processo crônico (Lima, 2007). 
Figura 1 - Tríade de Keyes.

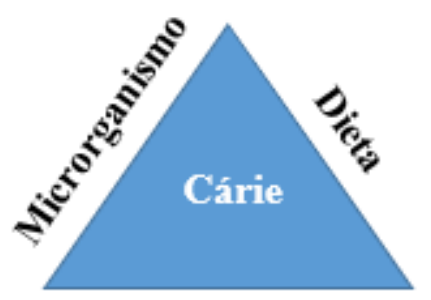

Dente susceptivel
Figura 2 - Fator etiológico tempo é acrescentado por Newbrum.

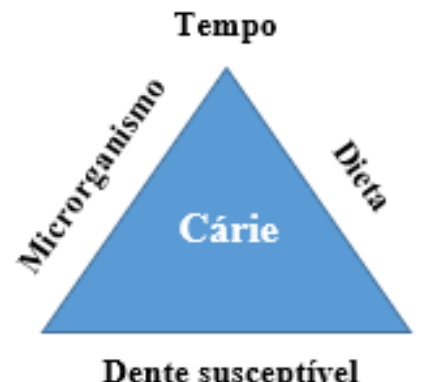

Fonte: Autores (2021).

Fonte: Autores (2021).

Ressalta-se que além dos fatores considerados fisiológicos, concernente à biodiversidade do organismo humano e das particularidades da cavidade bucal. Ainda deve-se levar em consideração as circunstancias socioeconómicos e comportamentais, que cumprem um papel importante na etiologia da doença, pois a cárie dentária inicia-se meramente como uma lesão do esmalte de causa local, progredindo à uma doença inflamatória, que por mais que apresente incidência baixa, não foi erradicada. Sendo esse processo determinado pelo estilo de vida do indivíduo, podendo se agravar com a negligência em higiene bucal e dieta a base de carboidratos fermentáveis (Campos et al., 2006; Lima, 2007).

\subsection{Potencial cariogênico de medicamentos, fármacos e drogas: mecanismos envolvidos}

Medicamentos são artigos elaborados a parti de fármacos, com efeito benéfico, seja este, de cura, profilático, paliativo ou diagnóstico. Os fármacos por sua vez, são produtos de estrutura química conhecida, que devido estudos frequentes, tem-se referenciado suas propriedades benéficas nos organismos. As drogas, no entanto, não apresentam estruturas física destrinchada, e podem ser definidas como qualquer substância, administradas a organismos vivos em quantidades tão pequenas que não ajam como alimentos, neles produzam alterações somáticas ou funcionais, com ou sem intenção benéfica (Bittencourt; Caponi; Maluf, 2013).

Os fármacos e medicamentos, termos utilizados a partir de agora como sinônimos, quando administradas de maneira repetida ou permanente, isto é, em patologias crônica, como nos casos de problemas cardíacos, alergias respiratórias (asma e bronquite), convulsões, doenças agudas recorrentes (amigdalite, otite, sinusite e rinite alérgica) e quimioterapia. Em pacientes específicos, geralmente pediatras, cujo as formas farmacêuticas destinadas a essa faixa etária, apresentam em sua composição algum tipo de açúcar para alterar propriedades organolépticas que facilitam sua administração. Podem levar a um resultado direto que resulta em hipofunção das glândulas salivares ou mudanças na microflora bucal, tendo como efeito secundário a cárie de medicamento (Goursand et al., 2006; Cabra, 2018; Bittencourt; Caponi; Maluf, 2013).

Outras situações devem ser evidenciadas. Como em pacientes que apresentam idade avançada, quer dizer, geriatras, que são polimedicados. Tendo em vista a prevalência de xerostomia correlacionada a polimedicação, e assim nessa modalidade induzindo à cárie. E que atualmente, considera a existência de mais de 400 fármacos xerostomizantes, distribuídos e vendidos nas redes de farmácias. Esse efeito prejudicando diretamente a ação tampão da saliva na prevenção de cáries (Abrantes, 2014).

E na perspectiva de abuso de drogas, um dos principais problemas de saúde e social do mundo. Sejam estas licitas ou ilícitas, podem provocar manifestações orais decorrentes do consumo. Devido sua ação direta na superfície dentaria, tendo efeito corrosivo, ou ocasionando perca de minerais essenciais. E a ação indireta e secundaria como efeitos de diminuição do fluxo salivar e imunossupressão. Todas essas repercussões, associado a negligência de higiene oral, podem se manifestar não somente como a cárie, mais também com alterações a nível de mucosas, ou ainda, celular (Jacob, 2014; Cabra, 2018). 
Descurtir-se-á agora detalhadamente os mecanismos envolvidos nas situações anteriormente citadas. Iniciando-se com as formas farmacêuticas pediátricas com concentrações elevadas de carboidratos fermentáveis. Dentre esses a sacarose é o principal destaque, essa serve de substrato para que os microrganismos da cavidade bucal formulem polissacarídeos extracelular e intracelular na placa dentária, e na produção de ácidos orgânicos, levando a grande atividade acidogênica com queda brusca do $\mathrm{pH}$ da placa dental. Atuando em auxílio a sobrevivência desses parasitos, pois ambientes ácidos favorecem seu desenvolvimento, e assim estes promovem a desmineralização do esmalte, podendo desencadear o processo de cárie (Freitas et al., 2016; Leal et al., 2015);

Além disto, formulações medicamentosas que oferecem a sacarose como edulcorante apresentam maior viscosidade, o que perturbar ou reduz o clearance salivar. Isso associado a capacidade xerostomizante dos princípios ativos das especialidades farmacêuticas podem acelerar o processo de desmineralização. No entanto ainda se apresenta controvérsias na literatura quanto ao potencial cariogênico dos medicamentos líquidos pediátricos. Devendo levar em consideração que para ocorrer esse processo, necessita-se da união de fatores que leva a tríade de Keyes (Alves et al, 2016; Lima, 2007).

Já em relação a hipofunção das glândulas salivares traz-se para facilitar a classificação farmacológica e terapêutica dos fármacos xerostomizantes, o sistema elaborado pela OMS (Organização Mundial da Saúde) chamado de ATC, sigla do inglês "Anatomical Therapeutic Chemical" referente a "Classificação terapêutica química e anatómica". Sendo os medicamentos divididos em 5 grupos distintos, de acordo com os sistemas de destino (local de ação), farmacodinâmica envolvida e as propriedades químicas e terapêuticas. A perturbação das glândulas salivares, neste caso a xerostomia, está em níveis de comprovação fortes ou discreto, em 9 dos 14 grupos existentes na ATC (Coimbra, 2009; Tayebati et al., 2017).

Podendo esse efeito adverso, ser descrito no grupo A- Trato digestivo e metabolismo: desordens do trato gastrointestinal (atropina e diciclomina); antieméticos (escopolamina e prometazina) e fármacos anti-obesidade. O efeito xerostomizante citado é decorrente das ações anti-colinérgicas, anti-histaminérgicos e anti-dopaminérgicos, mecanismos correspondentes as especialidades, de cada classe farmacológica. Grupo C- Aparelho cardiovascular: anti hipertensores (monoxidina, rilmenidina e clonidina); bloqueadores dos canais de cálcio (verapamil e isradipina); beta bloqueadores (timolol, metoprolol, bisoprolol e o atenolol) e os diuréticos (furosemida e bendroflumetiazida). Efeito decorrente da ação farmacológica em sítios, como receptores $\alpha-2$ adrenérgicos, $\beta$-1 adrenérgicos, canais de cálcio tipo L e transporte de íons como o sódio ( $\mathrm{Na}+$ ) e cloreto (Cl-) (Alegrias, 2019).

O grupo G envolve o Aparelho geniturinário e hormônios sexuais (oxibutina, propiverina, solifenacina, tolterodina, darifenacina, fesoterodina, cloreto de tróspio, tamsulosina e terazosina). Neste caso a xerostomia é resultado do bloqueio de receptores muscarínicos e também dos receptores $\alpha-1$ adrenérgicos (Bretas et al., 2008).

Grupo J estão os anti-infeciosos para uso sistémico: antirretrovirais, sendo três subclasses de destaque, os inibidores nucleosídeos da transcriptase reversa (didanosina, emtricitabina e lamivudina), inibidores não-nucleosídeos da transcriptase reversa (efavirenz, etravirine e nevirapina), e inibidores das proteases (darunavir, indinavir, nelfinavir, ritonavir e saquinavir). A xerostomia ocorre, pois, esses fármacos induzem acumulação atípica de gordura na glândula parótida, com posterior aumento de tamanho. Essa alteração fomentam a redução do fluxo salivar. Outros agentes retrovirais como o raltegravir também pode induzir esse feito (Pires, 2016; Tayebati et al., 2017; Alegrias, 2019;).

No Grupo L, os agentes anti-neoplásicos (bevacizumab, everolimo, tensilorimo, sorafenibe, dacomitinibe e nivolumab) e imunomodeladores (fármacos $\alpha 2$-interferon, usado na terapia da hepatite $\mathrm{C}$ e a interleucina-2), não apresentam mecanismo elucidado para observação da xerostomia, no entanto o efeito colateral é citado recorrentemente nas literaturas, pela sua utilização. O Grupo M, sistema músculo-esquelético (alendronato, baclofeno, tinazidina e a ciclobenzoprina) esse efeito adverso é registrado em ensaios randomizados controlados, e não apresentam mecanismo elucidado, mais traz referência 
as suas ações nos sistemas do metabolismo do cálcio, ácido gama-aminobutírico e glutamato, e ações antagônicas em receptores histamínicos, muscarínicos e a2-adrenérgicos (Bittencourt; Caponi; Maluf, 2013; Alegrias, 2019).

Fármacos do grupo N, sistema nervoso, são reagrupados em subgrupos: analgésicos, anticonvulsivantes, anti parkinsónicos, psicoanalépticos, psicolépticos, e diferentes fármacos empregados no sistema nervoso em situações de dependência. Assim no grupo dos analgésicos destaca-se os relacionados com os receptores opioides, cujo o efeito xerostomizante, foi evidenciado através de ensaios clínicos. Podendo citar como exemplos de medicamentos, a morfina, o butarfanol, o tapentadol, a buprenorfina e a dihidrocodeina (Tayebati et al., 2017)

No caso dos antiepilépticos a xerostomia é evidenciada pela limitada liberação de neurotransmissores, efeito ocasionada por diversos fármacos. Os neurotransmissores de destaque são, glutamato, noradrenalina, serotonina e dopamina. E o medicamento mais evidentemente reportado com esse efeito colateral, em literatura, é a carbamazepina. Há registros ainda de fármacos análogos do Gaba e antidepressivos utilizados como coadjuvantes nessas terapias (Abrantes, 2014).

Já em vista ao tratamento do Parkinson, o medicamento rotigotina, que interfere no sistema dopaminérgico, é evidenciado como xerostomizante em estudos randomizados. Por sua vez os psicoanalépticos, fármacos com alto potencial xerostomizante, e que apresentam mecanismos muito variáveis que envolvem bloqueio da recaptação de inúmeros neurotransmissores. Os antidepressivos são os medicamentos de relevância nesse grupo, os tricíclicos (imipramina, amitriptilina e nortriptilina) com maior incidência de indução de xerostomia, e os inibidores seletivos da recaptação de serotonina (fluoxetina, citalopram, paroxetina, setralina e excitalopram) com menor. Além é claro dos fármacos para tratamento de déficit de atenção ou hiperatividade (metilfenidato, o dexametilfenidato e lisdexanfetamina) (Bittencourt; Caponi; Maluf, 2013; Cabral, 2018).

E os psicolépticos, cujo efeito se dar fundamentalmente pelo aumento da interação do neurotransmissor Gaba pelos seus receptores, bloqueio de receptores colinérgicos do tipo muscarínicos, e adrenérgicos do tipo alfa1 e alfa2. É a classe de maior evidencia de indução de xerostomia, podendo citar como medicamentos desse grupo a aripiprazol, clorpromazina, clozapina, loxapina, litio, olanzapina, perfenazina, paliperidona, quetiapina, risperidona, zolpidem, ziprasidona, dentre outras (Abrantes, 2014).

Entrando agora no Grupo R, o sistema respiratório, os fármacos de notoriedade são os anti-histamínicos, cujo efeito xerostomizante se procede, não apenas pelo bloqueio de receptores de histamina, mais também pela capacidade desses fármacos de bloquear receptores muscarínicos do tipo M1 e M3. A doxilamina apresente fortes dados científicos desse efeito. Mais deve se considerar que anti-histamínicos mais modernos já apresentam modificações químicas que reduzem significativamente o efeito de xerostomia. Além destes os broncodilatadores bloqueadores de receptores muscarínicos como o ipratrópio e tiotrópio, de longa duração, podem ter como efeito adverso a xerostomia (Tayebati et al., 2017; Alegrias, 2019).

Por último, porém, não menos importante, o Grupo $\mathbf{S}$ que envolvem órgãos sensoriais. De ressalto fármacos de uso oftalmológico, como a atropina e a bromonidina, a primeira antagonista potente de receptores colinérgicos, e a segunda agonista dos receptores $\alpha 2$-adrenérgicos. Ambas podem ter como efeitos adversos a xerostomia (Tayebati et al., 2017; Alegrias, 2019).

É importante ressaltar que nenhuma das drogas anteriormente citadas está diretamente relacionada a cárie de medicamento. A relação aqui proposta é observar a importância do efeito tampão da saliva na profilaxia da cárie. Visto isso pode-se propor que qualquer fármaco ou medicamento indutora de xerostomia, em pacientes susceptíveis, com condições especificas, e a longo prazo de exposição, podem a vim a desenvolver essa patologia (Bretas et al., 2008).

Tendo em mente a ligação de prevenção da cárie com a capacidade da saliva de reduzir a acidez da cavidade oral e consequentemente a perda de tecido mineralizado, e ao fim à erosão no dente (Bretas et al., 2008). 
Em relações as drogas, classifica-se para melhor entendimento, em drogas licitas e ilícitas. As primeiras, o comercio é permitido por lei, em outras palavras legal. A segunda classe sua comercialização é proibida pela justiça. A relação do consumo dessas substâncias e as manifestações orais depende principalmente do consumo de forma abusiva, e supera o aspecto da cárie. Sendo bastante prejudicial para a cavidade bucal (Bittencourt; Caponi; Maluf, 2013).

Entres as drogas licitas cita-se o álcool (etanol $-\mathrm{C}_{2} \mathrm{H}_{5} \mathrm{OH}$ ) e o tabaco, esse último destaca-se pela presença de nicotina. O álcool é uma das drogas mais consumidas em todo mundo, e o seu abuso implica em efeitos deletério quando a saúde oral, além do surgimento de cáries, o prejuízo, em contraste ao alto consumo, ocasiona irritação da mucosa oral, e risco de gengivites, periodontites e carcinoma espinocelular (câncer bucal mais comum). O mecanismo pelo qual o etanol leva a esses efeitos maléficos estão relacionados a alta capacidade de diminuição do fluxo salivar, ocorrendo um aumento do índice de cáries, principalmente pela redução da capacidade tampão da saliva e consequente incapacidade de remoção mecânica do biofilme. Além disso, o paciente tende a apresentar inflamação constante da gengiva, o que leva a formação de bolsas periodontais e sangramento, aumentando maior detrimento (Spezzia, 2018).

A nicotina, presente no tabaco, planta da espécie Nicotiana tabacun, é a mais utilizada em forma de cigarro. O mecanismo pelo qual a nicotina exerce sua ação maléfica a cavidade oral está diretamente relacionada a sua capacidade de redução da resposta imunológica dos usuários. O que os torna propensos não só a doenças orais, como também sistêmicas. Além da cárie, o consumo a longo prazo pode ocasionar: gengivite, bolsas periodontais, perdas dentárias, halitose e câncer bucal (Marques et al., 2015).

Se tratando de drogas ilícitas, o escopo é imenso, portanto, esse artigo citará as drogas ilícitas mais comuns no mundo e as, mas citadas em literatura, ou seja, anfetamina, cocaína maconha e ecstasy. As anfetaminas são drogas sintéticas, cujo mecanismo de ação para seus efeitos alucinógenos e euforizantes envolve a estimulação do Sistema Nervoso Central, uma vez que inibe a recapacitarão de neurotransmissores importes para as sinapses. Entre as principais manifestações orais associados ao consumo de anfetaminas encontra-se a xerostomia, doença periodontais e cáries rampantes (destruição coronária rápida e bruxismo) (Marques et al., 2015).

A maconha, é uma planta do gênero Cannabis, que apresenta variedades de espécies. A mais conhecida em tono mundo é a Cannabis sativa. O mecanismo citado em bibliografias para exercer efeitos maléficos a cavidade bucal se faz referente a supressão da resposta imunológica, a nível humoral e celular. Além da xerostomia pelo seu consumo. A cárie é a principal manifestação oral pelo seu abuso, mais não o único, citando ainda periodontites, estomatite e cândidose (Spezzia, 2018).

Já a cocaína, uma substância obtida a partir da extração de folhas da planta Erythroxylon coca, é uma droga de alta toxidade para o sistema orgânico, sendo seus prejuízos incalculáveis. Na mucosa oral provoca efeitos vasoconstritores, e a longo prazo sua aspiração resulta em necrose e perfuração do septo nasal. A cocaína e seus derivados, crack e óxi, traz efeitos como gengivite ulcerativa, necrose aguda, periodontite avançada e lacerações gengivais. Essas lesões elevam o índice de cáries, cândidose e bruxismo. Esse resultado também se correlaciona a alta quantidade de produtos corrosivos e/ou irritantes presentes em seus derivados, substâncias como gasolina, querosene, ácido clorídrico e solda caustica, são adicionadas para sua produção e barateamento do produto (Marques et al., 2015; Spezzia, 2018).

Por último, cita-se o ecstasy, cujo princípio ativo de suas ações é atribuído ao constituinte químico 3,4metilenodioximetanfetamina ou MDMA. Os efeitos são variáveis, mais o mecanismo pelo qual ocasiona a cárie é semelhante aos já citados, tendo relação com a xerostomia. É importante ressaltar que ainda pode levar a outras manifestações orais do tipo: periodontites, sensibilidade dentária, bruxismo, parestesia do nervo, edemas e úlceras (Marques et al., 2015; Spezzia, 2018). 
E de maneira geral, os dependentes químicos normalmente apresentam negligência da higiene oral, outro fator que favorece a colonização por microrganismo patogênico que formarão placa dentária. E muitas vezes a utilização dessas drogas aumentam o limiar da dor, mascarando a sintomatologia dolorosa, agravando o quadro clínico do paciente (Marques et al., 2015).

\subsection{Prevenção do desenvolvimento da cárie de medicamento}

Considerando que a doença cárie é passível de prevenção, sendo principalmente aquelas induzidas por medicamentos, fármacos e drogas, apresenta-se as medidas fundamentais, profiláticas para o problema. Tendo principalmente a informação repassada por profissionais capacitados para validação dos episódios. Médicos, especialmente pediatras e geriatras, dentistas e farmacêuticos, se encontram em condições ideais para alertar, informar e orientar sobre os riscos inerentes ao uso contínuo, de medicamentos que apresentam em sua formulação algum tipo de açúcar, sobre polifarmácia, abuso de substâncias licitas e ilícitas, e seus efeitos adversos sobre a cavidade oral (Neves; Pierro \& Maia, 2007).

Além de tudo, existem no mercado formulações a base de excipientes, hipoacidogênico, como o sorbitol, sendo alternativa para especialidades farmacêuticas com alta concentrações de açúcares fermentáveis. Disponibilizando medicamentos alternativos mais acessíveis e menos prejudiciais (Leal et al., 2015).

A orientação de hábitos de higiene bucal adequadas para pacientes, pelo clínico dentista. E o aconselhamento medicamentoso realizado pelo farmacêutico quando o cliente obtém o produto, são exemplos de ações que reduzem significativamente o efeito adverso cárie de medicamento (Cabral, 2018).

E se tratando de dependentes químicos, o papel do cirurgião dentista vai muito além da identificação desses pacientes. A atenção é imprescindível e diferenciada, levando sempre em consideração, a capacidade do paciente em se comprometer com o tratamento. Isso com auxílio de especialidade médica e psiquiatra, visando o acompanhamento multidisciplinar, pode reverter por completo o quadro de enfermidade do usuário (Marques et al., 2015; Alves et al.,2016).

\section{Considerações Finais}

Sugere-se que os medicamentos, fármacos e drogas apresentam potencial cariogênico seja pela presença de açúcares fermentáveis na sua composição, ou pelo seu efeito adverso, xerostomia, e ainda ação corrosiva direta sobre os dentes. E que esta cárie só afetara os pacientes em condições especificas e organismos susceptíveis. Sendo um problema severo, mais que possue prevenção, tendo a relação direta ao auxílio dos pacientes por uma equipe multidisciplinar que envolve médicos, dentistas e farmacêuticos (Sousa et al., 2010; Alves et al.,2016).

Ainda pode-se observar que a cárie de medicamentos apresenta característica rampante, podendo atingir incisivos e molares, e que os mecanismos das substâncias para ocasionar esse problema são bastante variáveis, dependo da classe, dose e dosagem e cronicidade de exposição. Sendo que a prevenção parece ser a estratégia mais adequada para controle da cárie de medicamento (Cabral, 2018).

\section{Referências}

Alves, V. F. et al. (2016). Efeito sobre a morfologia do esmalte dental e análise físico-química de medicamentos utilizados por pacientes pediátricos com paralisia cerebral. Revista de Odontologia da UNESP, 45(4), 201-205.

Alegrias, P. M. P. (2019). Planeamento de um estudo da xerostomia em indivíduos saudáveis e polimedicados com aplicação de um estimulador salivar. 2019. Dissertação (Mestrado em Medicina Dentária) - Instituto Universitário Egas Moniz.

Abrantes, J. P. M. de. (2014). A xerostomia no idoso. Dissertação (Mestrado integrado em medicina dentária) - Universidade Porto.

Bretas, L. P. et al. (2008). Fluxo salivar e capacidade tamponante da saliva como indicadores de susceptibilidade à doença cárie. Pesq Bras Odontoped Clin Integr, 8 (3), 289-293. 
Bittencourt, S. C.; Caponi, S., \& Maluf, S. (2013) Farmacologia no século XX: a ciência dos medicamentos a partir da análise do livro de Goodman e Gilman. História, Ciências, Saúde, 20 (2), 499-519.

Campos, J. Á. D. B. et al. (2006) Correlação entre a prevalência de cárie e a utilização de medicamentos em pacientes com necessidades especiais institucionalizados e não institucionalizados. Salusvita, 25 (1), 35-42.

Coimbra, F. Xerostomia. Etiologia e Tratamento. (2009). Revista Portuguesa de Estomatologia, Medicina Dentária e Cirurgia Maxilofacial, 50 (3).

Cabral, B. M. M. (2018). Importância do farmacêutico no diagnóstico e tratamento da xerostomia. Dissertação. Universidade Lusófona de Humanidades e Tecnologias - Escola de Ciências e Tecnologias da Saúde, Lisboa.

Freitas, A. I, J. (2015) A abordagem do médico dentista ao doente diabético. 2015. Dissertação (Mestrado em Medicina Dentária) - Instituto Superior de Ciências da Saúde Egas Moniz.

Freitas, M. P. R. et al. (2016). Conhecimento dos pediatras sobre os efeitos dos medicamentos na cavidade bucal. Arquivos Catarinenses de Medicina, 45 (4), $67-77$.

Goursand, D. et al. (2006). Sequelas bucais em crianças submetidas à terapia antineoplásica: causas e definição do papel do cirurgião dentista. Arquivos em Odontologia, 42 (3), 180-189.

Gomes, Viviane Elisângela \& Silva, Débora Dias da. (2010). A importância do controle de placa dental na clínica odontológica. Arquivos em Odontologia, 461 (1).

Jacob, S.; \& Iwasaki, K. K. (2014). A influência do antibiótico na cárie infantil. Brazilian Journal of Surgery and Clinical Research -BJSCR, 8(2), 68-74.

Leal, W. M. S. et al. (2015). Entendendo a relação entre medicamentos de uso pediátrico e cárie dentária. Revista de Pediatria SOPERJ, 15(2), 16-21.

Lima, J, E, O. (2007). Cárie dentária: um novo conceito. R. Dental Press Ortodon Ortop Facial, 12 (6), 119-130.

Marques, L, A, R, V. et al. (2015) Abuso de drogas e suas consequências na saúde oral: uma revisão de literatura. Arquivo Brasileiro de Odontologia, 11 (1).

Neves, B. G.; Pierro, V. S. S. \& Maia, L. C. (2007) Percepções e atitudes de responsáveis por crianças frente ao uso de medicamentos infantis e sua relação com cárie e erosão dentária. Ciência \& Saúde Coletiva, 12 (5), 1295-1300.

Passos, I. A; Freitas, C. H. S. M.; \& Sampaio, F. C. (2008) Potencial cariogênico de medicamentos pediátricos - papel na etiologia da cárie dentária. Revista do Instituto de Ciências da Saúde, 125-129.

Pires, W. R. (2016). Efeito da antibioticoterapia sistêmica com amoxicilina e tetraciclina no processo de reparo da injúria de luxação extrusiva. Dissertação (Mestrado em Odontologia) Universidade Estadual Paulista, Faculdade de Odontologia de Araçatuba.

Silva, A. S. M. da. (2016). Microbioma oral: o seu papel na saúde e na doença. Dissertação (Mestre em ciências farmacêuticas) - Universidade Lusófona de Humanidades e Tecnologias- Escola de Ciências e Tecnologias da Saúde.

Sousa, R. I. M. et al. (2010). Potencial erosivo e cariogênico de anti-histamínicos de uso infantil. Revista da Faculdade de Odontologia, 15 (3), $255-260$.

Spezzia, S. (2018). Repercussões bucais do uso de drogas na adolescência. Rev Ciênc Med. 27 (2), 93-100.

Tayebati, S. K., et al. (2017). Identification of World Health Organisation ship's medicine chest contents by Anatomical Therapeutic Chemical (ATC) classification codes. International Maritime Health, 68 (1), 39-45. 